\title{
PAPER \\ Performance Analysis of Hermite-Symmetric Subcarrier Coding for OFDM Systems over Fading Channels
}

\author{
Fumihito SASAMORI $^{\dagger \mathrm{a})}$ and Shiro HANDA ${ }^{\dagger}$, Members
}

\begin{abstract}
SUMMARY Orthogonal frequency division multiplexing (OFDM) has great advantages such as high spectrum efficiency and robustness against multipath fading. In order to enhance the advantages, an Hermitesymmetric subcarrier coding for OFDM, which is used for transmission systems like the asymmetric digital subscriber line (ADSL) and multiband OFDM in ultra-wideband (UWB) communications, is very attractive. The subcarrier coding can force the imaginary part of the OFDM signal to be zero, then another data sequence can be simultaneously transmitted in the quadrature channel. In order to theoretically verify the effectiveness of the Hermite-symmetric subcarrier coding in wireless OFDM (HC-OFDM) systems, we derive closed-form equations for bit error rate (BER) and throughput over fading channels. Our analytical results can theoretically indicate that the HC-OFDM systems achieve the improvement of the performances owing to the effect of the subcarrier coding.

key words: OFDM, Hermite-symmetric coding, maximum ratio combining, frequency correlation, closed-form equation
\end{abstract}

\section{Introduction}

Orthogonal frequency division multiplexing (OFDM) [1], [2], which is applied to wireless LAN, digital terrestrial TV broadcasting, terrestrial mobile communication and so on, has great advantages such as high spectrum efficiency and robustness against multipath fading. In order to enhance the advantages, many elemental wireless technologies have been combined with OFDM, for example, space-time (or space-frequency) coding for diversity [3]-[5], adaptive modulation [6], [7], forward error correction (FEC) [8]-[10] and multi-input multi-output (MIMO) [11], [12].

We have been focused on a simple kind of coded OFDM with a repetition code on the frequency domain, which is based on IEEE 802.16 protocols [13], [14]. The repetition coded OFDM (RC-OFDM) scheme brings many good results, such as inter-carrier interference (ICI) cancellation [15], [16], blind channel estimation [17], and bit error rate (BER) improvement owing to diversity gain [16], [18]. In [18], we have introduced the RC-OFDM systems employing diversity combining and higher-level modulation with such a simple structure as one pair of transmit/receive antennas. All subcarriers of OFDM are divided into two regions (low and high subcarrier frequencies), then PSK or QAM symbols are duplicated with half the subcarriers spacing. Due to both double bit energy and frequency diversity gain, BER and throughput performances are much improved

Manuscript received January 24, 2012.

Manuscript revised July 13, 2012.

$\dagger$ The authors are with the Faculty of Engineering, Shinshu University, Nagano-shi, 380-8553 Japan.

a)E-mail: fsasa@shinshu-u.ac.jp

DOI: 10.1587/transfun.E95.A.2461 as compared with those in the general OFDM systems on the conditions of the same carrier power and the same bit rate. Moreover, we have derived simple closed-form equations for BER and throughput to theoretically verify the effectiveness of the repetition coding.

On the other hand, characteristics of the Fourier transform can be also utilized to enhance the advantages of OFDM, since OFDM modulation and demodulation are conveniently performed by fast Fourier transform (FFT) and its inverse transform (IFFT) operations. For example, the half-symbol transmission techniques are very attractive in that the duration of the guard interval can be virtually and flexibly extended to half the OFDM symbol duration for large multipath delays [19], [20]. Moreover, an Hermitesymmetric subcarrier coding for OFDM, which is used for transmission systems like the asymmetric digital subscriber line (ADSL) [21], [22] and low data rate mode of multiband OFDM (MB-OFDM) in ultra-wideband (UWB) communications [23], is also very attractive. The subcarrier coding is based on the fact that the Fourier transform of real-valued input without imaginary part becomes Hermitesymmetric. The imaginary part of the OFDM signal can be forced to be zero, then another data sequence can be simultaneously transmitted in the quadrature channel. In high data rate mode of the MB-OFDM, convolutionally encoded bits are modulated by QPSK or dual carrier modulation (DCM) [24]. The DCM symbol with 16QAM constellation is mapped onto two individual subcarieers with 50 subcarriers separation (without Hermite-symmetry) and Viterbi decoder is used in the receiver, which is classified into the RC-OFDM.

In this paper, in order to theoretically verify the effectiveness of a combination of the repetition coding, the characteristic of the Fourier transform, and the simultaneous transmission of the two data sequences with the aid of a phase compensator over fading channels [25], we derive closed-form equations for BER and throughput of wireless OFDM systems employing the Hermite-symmetric subcarrier coding, which we call 'HC-OFDM' hereafter. Because of the dual channel transmission, higher-level modulation to keep the same bit rate like the RC-OFDM systems is unnecessary, while double carrier power is consumed. Therefore, the signal power per channel in the HC-OFDM systems should be reduced by half in order to keep the same condition. But, by enhancing the analytical method in [18], it can be theoretically and quantitatively indicated that the HC-OFDM systems achieve the improvement of BER and 
throughput performances owing to the frequency diversity gain and the dual channel transmission. The derived equation of throughput can be also utilized for verifying the advantage of the HC-OFDM systems when applying the adaptive modulation.

From the standpoint of a simple transmit diversity technique to easily provide the same diversity gain as maximum ratio combining (MRC) with only one receive antenna, the space-time (or space-frequency) coding for OFDM with multiple transmit antennas is attractive [4], [5]. Those are classified into space diversity scheme, since the channel coefficients of successive time slots (or subcarriers) are almost the same. On the other hand, the Hermite-symmetric subcarrier coding, which is classified into frequency diversity scheme, is also attractive in that only one pair of transmit/receive antennas is required in spite of the limited diversity gain which depends on the frequency selectivity.

The remainder of this paper is organized as follows. A principle of the Hermite-symmetric subcarrier coding and a system model of the HC-OFDM systems are described in Sect. 2. Closed-form equations for BER and throughput are derived for the purpose of theoretically verifying the effectiveness of the HC-OFDM systems over fading channels in Sect. 3, and the performances are evaluated in Sect. 4. An additional performance evaluation with error-correcting code is conducted in Sect. 5, and the paper is concluded in Sect. 6.

\section{Hermite-Symmetric Subcarrier Coding}

2.1 Principle of Subcarrier Coding with Hermite Symmetry

We briefly introduce a principle of the subcarrier coding with Hermite symmetry [22]. Complex baseband OFDM symbols $\left\{s_{n}\right\}$ using $N_{d}$-point inverse discrete Fourier transform (IDFT) are generally expressed as

$$
s_{n}=\sum_{i=0}^{N_{d}-1} S_{i} \exp \left(j 2 \pi \frac{i n}{N_{d}}\right),
$$

where $S_{i}$ is a complex symbol in the $i$-th subcarrier. The Hermite-symmetric subcarrier coding is based on the fact that the Fourier transform of real-valued input without imaginary part becomes Hermite-symmetric. Concretely speaking, if $\left\{S_{i}\right\}$ have Hermite symmetry such as

$$
S_{N_{d}-i}=S_{i}^{*}, \quad\left(i=1,2, \cdots, \frac{N_{d}}{2}-1\right),
$$

the OFDM symbols $\left\{s_{n}\right\}$ become real numbers. That is, we can rewrite (1) as follows:

$$
\begin{aligned}
s_{n}= & S_{0}+\sum_{i=1}^{\frac{N_{d}}{2}-1} S_{i} \exp \left(j 2 \pi \frac{i n}{N_{d}}\right)+S_{\frac{N_{d}}{2}} \cos (\pi n) \\
& +\sum_{i=1}^{\frac{N_{d}}{2}-1} S_{N_{d}-i} \exp \left\{j 2 \pi \frac{\left(N_{d}-i\right) n}{N_{d}}\right\} .
\end{aligned}
$$

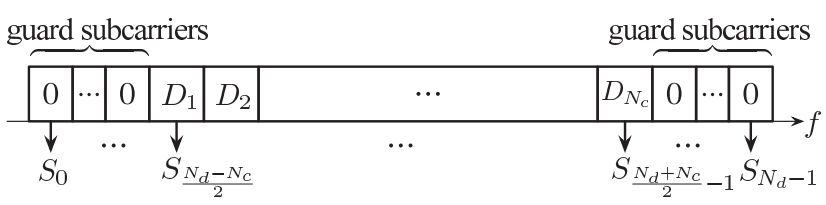

(a) OFDM

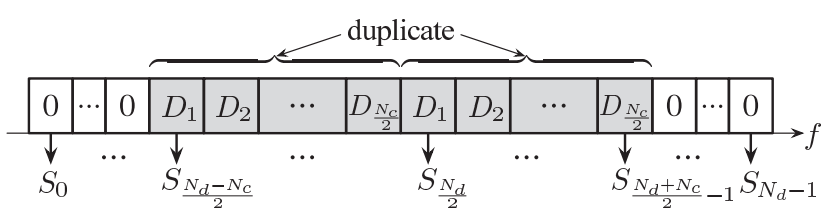

(b) repetition coded OFDM (RC-OFDM)

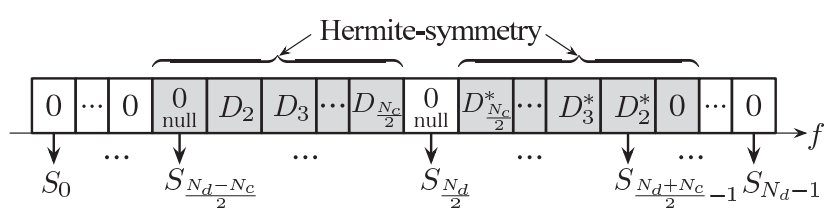

(c) Hermite-symmetric coded OFDM (HC-OFDM)

Fig. 1 Concept of subcarrier coding.

Now we define the complex symbols $\left\{S_{i}\right\}$ as

$$
S_{i} \equiv R_{i}+j I_{i}
$$

where both $\left\{R_{i}\right\}$ and $\left\{I_{i}\right\}$ are real numbers, then $\left\{s_{n}\right\}$ can be expressed as

$$
\begin{aligned}
s_{n}= & S_{0}+S_{\frac{N_{d}}{2}} \cos (\pi n) \\
& +2 \sum_{i=1}^{\frac{N_{d}}{2}-1}\left\{R_{i} \cos \left(2 \pi \frac{i n}{N_{d}}\right)-I_{i} \sin \left(2 \pi \frac{i n}{N_{d}}\right)\right\} .
\end{aligned}
$$

Therefore, if $S_{0}$ and $S_{N_{d} / 2}$ are real numbers, imaginary part of the OFDM symbols $\left\{s_{n}\right\}$ can be forced into zero. It should be noted that the average power of the OFDM symbols $\left\{s_{n}\right\}$ remains constant whether the complex symbols $\left\{S_{i}\right\}$ have Hermite symmetry or not.

Figure 1 shows a concept of the subcarrier coding, where $N_{c}$ is the number of subcarriers. In general OFDM systems, PSK or QAM symbols $\left\{D_{j}\right\}_{j=1,2, \cdots, N_{c}}$ with guard subcarriers are input into IDFT (IFFT) ports $\left\{S_{i}\right\}$ like Fig. 1(a). Figure 1(b) shows the repetition coding scheme [18]. The symbols $\left\{D_{j}\right\}_{j=1,2, \cdots, N_{c} / 2}$ are duplicated with $N_{c} / 2$ subcarriers spacing at a transmitter and the two symbols are combined with $\mathrm{MRC}$ at a receiver to acquire the frequency diversity gain. The information bit rate is reduced by half owing to the duplication, then the higher-level modulation should be considered in the RC-OFDM systems to keep the same bit rate. On the other hand, in the Hermite-symmetric coding, the symbols $\left\{D_{j}\right\}_{j=2,3, \cdots, N_{c} / 2}$ are duplicated and conjugated, then those symbols are input into IDFT (IFFT) ports $\left\{S_{i}\right\}$ with Hermite symmetry like Fig. 1(c). In order to keep the symmetry, the following rules are necessary:

- $S_{0}$ in (5) should be zero because of a guard subcarrier.

- A real-valued BPSK symbol can be input to $S_{N_{d} / 2}$ in 


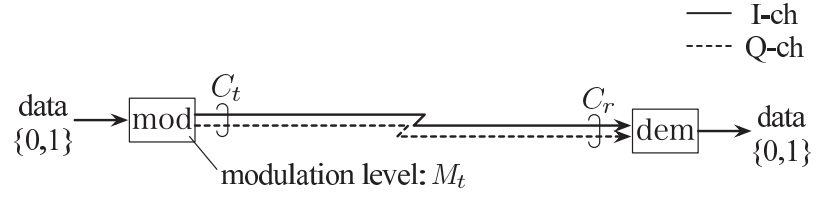

(a) OFDM

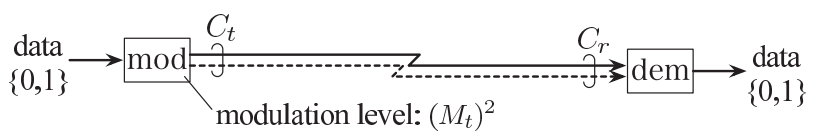

(b) RC-OFDM

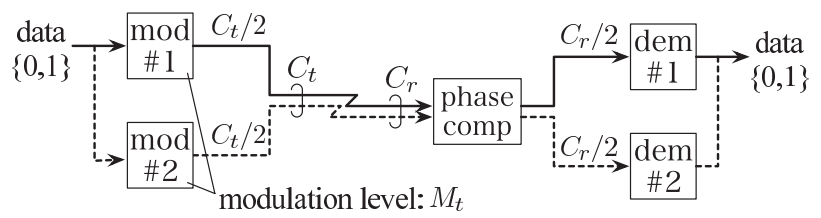

(c) HC-OFDM

Fig. 2 Comparison between three OFDM systems.

(5). But, for simplicity, $S_{N_{d} / 2}$ is set to zero (nullsubcarrier) in this paper.

- $S_{\left(N_{d}-N_{c}\right) / 2}$ should be zero (null-subcarrier), because $S_{\left(N_{d}+N_{c}\right) / 2}$ which is the symmetric pair of $S_{\left(N_{d}-N_{c}\right) / 2}$ is one of the guard subcarriers.

The information bit rate is reduced by more than half (precisely speaking, $\left(1 / 2-1 / N_{c}\right)$ times reduction). But, if the coherent detection is ideal, another symbols $\left\{D_{j}^{\prime}\right\}_{j=2,3, \cdots, N_{c} / 2}$ can be transmitted simultaneously in the quadrature channel, that is, the imaginary-part of the OFDM signal in (5).

\subsection{System Model}

Figure 2 shows the comparison between three OFDM systems on the conditions of the same carrier power and the same bit rate, where $C_{t}$ and $C_{r}$ are transmitted and received carrier powers, respectively, and $M_{t}$ is the modulation level of the general OFDM systems. $C_{t}, C_{r}$ and $M_{t}$ are assumed to be fixed in the three systems. As explained above, the bit rate per symbol $R$ in $M$-PSK or $M$-QAM can be expressed as

$$
R= \begin{cases}\log _{2} M & \text { (for OFDM) } \\ \frac{1}{2} \log _{2} M & \text { (for RC-OFDM) } \\ \left(1-\frac{2}{N_{c}}\right) \log _{2} M & \text { (for HC-OFDM). }\end{cases}
$$

In order to keep the same bit rate, the modulation level $M$ must be

$$
M= \begin{cases}M_{t} & (\text { for OFDM, HC-OFDM }) \\ \left(M_{t}\right)^{2} & (\text { for RC-OFDM })\end{cases}
$$

then the power efficiency in digital-modulation is degraded in the RC-OFDM systems. In the HC-OFDM systems, owing to the simultaneous transmission of two data sequences, almost the same bit rate per symbol $R$ can be maintained in the case of large subcarrier size $N_{c}$. Therefore, the modulation level and its power efficiency in the HC-OFDM systems are the same as those in the OFDM systems. But twice the digital signal processings of modulation/demodulation are necessary to send two data sequences, and a phase compensator is indispensable to correct the imbalance between IQ channels caused by carrier frequency offset and fading [25].

As for the carrier power consumption, since the average powers of $\left\{s_{n}\right\}$ in (1) and (5) are the same, the transmission with both IQ channels in the HC-OFDM systems causes to consume double carrier power. Therefore, the signal power per channel should be reduced by half in order to keep the same condition as shown in Fig. 2(c). Though the symbol energy for demodulation at a receiver is also reduced by half, our analytical results mentioned later can theoretically indicate that the HC-OFDM systems achieve the improvement of BER and throughput performances.

Figure 3 shows a block diagram of HC-OFDM systems. At a transmitter, a binary data sequence is converted to digital-modulated symbols $\left\{D_{j}\right\}_{j=2,3, \cdots, N_{c} / 2}$, which are parallelized by serial-to-parallel (S/P) conversion and are coded by the Hermite-symmetric subcarrier coding scheme mentioned above. The coded symbols $\left\{S_{i}\right\}_{i=0,1, \cdots, N_{d}-1}$ are converted to discrete OFDM symbols $\left\{s_{n}\right\}_{n=0,1, \cdots, N_{d}-1}$ by OFDM modulation (IFFT). After parallel-to-serial (P/S) conversion, guard interval (GI) insertion and digital-to-analog (D/A) conversion, generated OFDM signal is transmitted with half the carrier power in the I channel. Another data sequence is modulated in the same way and is simultaneously transmitted in the $\mathrm{Q}$ channel. The OFDM signal is subjected to Rayleigh fading and is added white Gaussian noise (AWGN). The fading channel is frequency-selective, but it is assumed that inter-symbol interference (ISI) caused by multipath delay spread can be completely avoided with the GI, which is longer than the maximum multipath delay. At a receiver, after analog-to-digital (A/D) conversion, the two OFDM symbols are compensated by a phase compensator to correct the IQ imbalance. A data sequence is demodulated by reversing the process of the modulation as follows: GI deletion, S/P conversion, OFDM demodulation (FFT), coherent detection, subcarrier decoding with $\mathrm{MRC}, \mathrm{P} / \mathrm{S}$ conversion and data decision. Since the OFDM signal is affected by multipath fading, the channel estimation is necessary for the purpose of phase compensation and coherent detection [25]-[27]. In order to theoretically evaluate the optimal advantage of the Hermite-symmetric subcarrier coding, the perfect channel estimation is assumed in this paper.

\section{Performance Analysis}

In order to verify the effectiveness of the Hermite-symmetric coding over fading channels, we will theoretically derive closed-form equations for BER and throughput in this section.

The average BERs with Gray-mapped $M$-PSK or $M$ QAM in the OFDM and the RC-OFDM systems over 

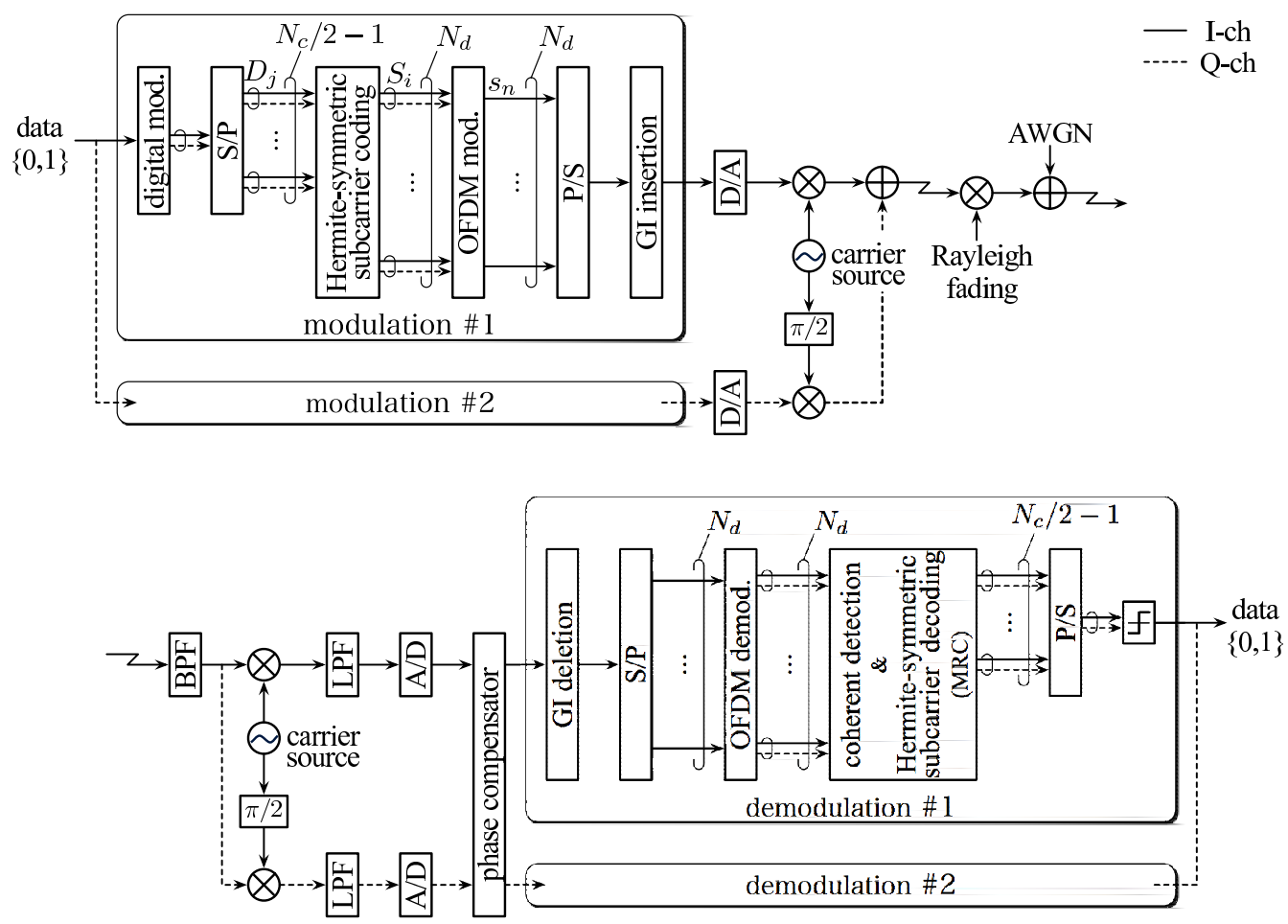

Fig. 3 Block diagram of HC-OFDM systems.

Rayleigh fading channels can be calculated by [18]

$$
\begin{aligned}
& \bar{P}_{b}=A_{i}\left(1-\frac{1}{\sqrt{1+\frac{1}{B_{i} \Gamma_{s}}}}\right) \text { (for OFDM), } \\
& \bar{P}_{b}=A_{i}\left[1-\frac{1}{2\left|\rho_{B}\left(\frac{N_{c}}{2}\right)\right|}\left\{\frac{1+\left|\rho_{B}\left(\frac{N_{c}}{2}\right)\right|}{\sqrt{1+\frac{1}{\left\{1+\left|\rho_{B}\left(\frac{N_{c}}{2}\right)\right|\right\} B_{i} \Gamma_{s}}}}\right.\right. \\
& \left.\left.-\frac{1-\left|\rho_{B}\left(\frac{N_{c}}{2}\right)\right|}{\sqrt{1+\frac{1}{\left\{1-\left|\rho_{B}\left(\frac{N_{c}}{2}\right)\right|\right\} B_{i} \Gamma_{s}}}}\right\}\right] \quad \text { (for RC-OFDM), } \\
& \Gamma_{s}=\frac{\Gamma}{1+\alpha} \text { (for OFDM, RC-OFDM), }
\end{aligned}
$$

where $\Gamma$ is average carrier-to-noise power ratio (CNR) of received signal, $\alpha$ is the ratio of GI duration $T_{g}$ to data symbol duration $T_{d}\left(\alpha=T_{g} / T_{d}\right)$, and then $\Gamma_{s}$ in (10) means the CNR after GI deletion. $\left|\rho_{B}(l)\right|$ is the absolute frequency correlation between symbols with $l$ subcarriers spacing, which can be derived from any discrete-time power delay profile model. The values of $A_{i}$ and $B_{i}\left(i=\log _{2} M\right)$ in each modulation are appeared in Table 1.

On the other hand, the BER in the HC-OFDM systems can be calculated by modifying (9). The subcarriers spacing between two symbols to be combined is always kept to $N_{c} / 2$ in the repetition coding in Fig. 1(b). But the subcarriers spacing between the symmetric pair of symbols varies as shown in Fig. 1(c), which results in varying the BER. Fig-
Table 1 Values of $A_{i}$ and $B_{i}$.

\begin{tabular}{c|c|c|c}
\hline \hline$i$ & Modulation & $A_{i}$ & $B_{i}$ \\
\hline \hline 1 & BPSK & $1 / 2$ & 1 \\
\hline 2 & QPSK & $1 / 2$ & $1 / 2$ \\
\hline 3 & 8PSK & $1 / 3$ & $\sin ^{2}(\pi / 8)$ \\
\hline 4 & 16QAM & $3 / 8$ & $1 / 10$ \\
\hline 6 & 64QAM & $7 / 24$ & $1 / 42$ \\
\hline 8 & 256QAM & $15 / 64$ & $1 / 170$ \\
\hline \hline
\end{tabular}

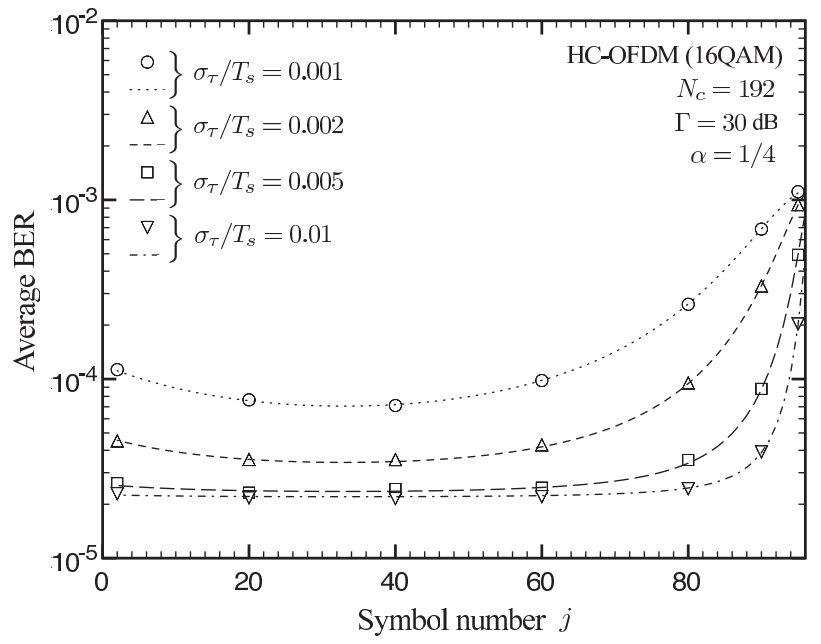

Fig. 4 Average BER of each digital-modulated symbol in HC-OFDM systems (16QAM, $N_{c}=192, \Gamma=30 \mathrm{~dB}, \alpha=1 / 4$ ).

ure 4 shows the average BER of each symbol $\left\{D_{j}\right\}_{j=2,3, \cdots, N_{c} / 2}$ in the case of 16QAM, $N_{c}=192, \Gamma=30 \mathrm{~dB}$ and $\alpha=1 / 4$, 
where simulation results are plotted with symbols and analytical results are with lines. It is found from the figure that the BER varies depending on the frequency correlation characteristics, which is shown in Fig. 3 of [18], at any rms delay spread $\sigma_{\tau}$ normalized by OFDM symbol duration $T_{s}$ $\left(=T_{d}+T_{g}\right)$. That is, the average BER is more degraded around the center of all subcarriers because of less frequency diversity gain. For the purpose of easy comparison, we calculate the BER in the HC-OFDM systems by averaging the BER in (9) as follows:

$$
\begin{array}{r}
\bar{P}_{b}=\frac{A_{i}}{\frac{N_{c}}{2}-1} \sum_{l=1}^{\frac{N_{c}}{2}-1}\left[1-\frac{1}{2\left|\rho_{B}(2 l)\right|}\left\{\frac{1+\left|\rho_{B}(2 l)\right|}{\sqrt{1+\frac{1}{\left\{1+\left|\rho_{B}(2 l)\right|\right\} B_{i} \Gamma_{s}}}}\right.\right. \\
\left.-\frac{1-\left|\rho_{B}(2 l)\right|}{\sqrt{1+\frac{1}{\left\{1-\left|\rho_{B}(2 l)\right| B_{i} \Gamma_{s}\right.}}}\right\} \text { (for HC-OFDM). }
\end{array}
$$

As shown in Fig. 2(c), only half the carrier power can be used for demodulation as compared with (10), then $\Gamma_{s}$ in (11) should be defined as

$$
\Gamma_{s}=\frac{\Gamma}{2(1+\alpha)}(\text { for HC-OFDM })
$$

As well as the RC-OFDM systems, full diversity gain cannot be achieved because of frequency correlation between subcarriers, but we will theoretically verify the advantage of the HC-OFDM systems despite half the carrier power and the imperfect diversity gain in the next section.

When the frequency spacing and/or the rms delay spread are very large, that is, when two symbols with MRC can be considered to be mutually independent, the maximum diversity gain (the minimum BER) can be achieved in the RC-OFDM and HC-OFDM systems. Then the BER becomes [18]

$$
\bar{P}_{b_{-} \min }=A_{i}\left\{1-\frac{1+\frac{3}{2 B_{i} \Gamma_{s}}}{\left(1+\frac{1}{B_{i} \Gamma_{s}}\right)^{\frac{3}{2}}}\right\} \text {. }
$$

Finally, the average throughput in terms of bits per second per Hertz can be calculated by

$$
\bar{S}=\frac{R\left(1-\bar{P}_{b}\right)^{D}}{1+\alpha},
$$

where $1 /(1+\alpha)$ means the loss by GI insertion and $D$ is a packet size (the number of bits per packet). It should be noted in the throughput analysis in the upper layer of the OSI model that the packet size is assumed to be set irrelevantly to the number of bits per OFDM symbol, and that an optimal bit interleaver is assumed to randomize burst bit errors.

We have been derived closed-form equations for BER and throughput in terms of CNR $\Gamma$, while the equations can be changed in terms of the energy per bit to noise power spectral density ratio $E_{b} / N_{0}$ by [28]

$$
\begin{aligned}
E_{b} / N_{0} & =\frac{\Gamma}{\log _{2} M} \\
& = \begin{cases}\frac{\Gamma}{\log _{2} M_{t}} & \text { (for OFDM, HC-OFDM) } \\
\frac{\Gamma}{2 \log _{2} M_{t}} & \text { (for RC-OFDM). }\end{cases}
\end{aligned}
$$

The evaluation in terms of CNR is useful in that the throughput performance comparison between systems with different bit rate when considering adaptive modulation [7], then we use CNR for the performance evaluation in the next section.

\section{Performance Evaluation}

We theoretically verify the advantages of the HC-OFDM systems as compared with the conventional ones. In order to justify the theoretical analysis in Sect. 3, performances obtained by Monte Carlo simulation are also evaluated. Table 2 shows the simulation parameters, and Table 3 shows the two cases of the subcarrier modulations to be compared on the same conditions. In the simulation, a tapped-delay line channel simulator is considered as the fading channel [29], and each channel tap is simulated using the Jakes' model [30]. An exponential decay power delay profile is assumed [31] in the multipath fading channel model.

Figures 5 and 6 show the average BER and throughput performances over Rayleigh fading channels when the subcarrier size $N_{c}=192$ and the normalized rms delay spread $\sigma_{\tau} / T_{s}=0.01$. Simulation results are plotted with symbols and analytical results by (8), (9), (11), (14) are with lines in the figures. In Fig. 5, the two kinds of performances are simultaneously shown as reference: in the case of HCOFDM with higher-level modulation in the I channel only, and in the case of HC-OFDM attaining the maximum diversity gain by (13). It is found from Figs. 5 and 6 that the HC-OFDM systems can outperform the conventional ones despite half the carrier power in (12). The performance of the HC-OFDM systems with higher-level modulation in the single channel is slightly degraded as compared with

Table 2 Simulation parameters.

\begin{tabular}{c|c}
\hline \hline Modulation & PSK, QAM \\
\hline Detection & Coherent (ideal) \\
\hline FFT point size $N_{d}$ & $64,128,256,512$ \\
\hline Subcarrier size $N_{c}$ & $48,96,192,384$ \\
\hline GI ratio $\alpha\left(=T_{g} / T_{d}\right)$ & $1 / 4$ \\
\hline Packet size $D$ & 100 \\
\hline Noise & AWGN \\
\hline Fading & Rayleigh fading \\
\hline Channel model & Exponential decay \\
\hline \hline
\end{tabular}

Table 3 Subcarrier modulations to be compared.

\begin{tabular}{c|c|c|c|c}
\hline \hline & Mod. level $M_{t}$ & OFDM & RC-OFDM & HC-OFDM \\
\hline case I & 4 & QPSK & 16QAM & QPSK \\
\hline case II & 16 & 16QAM & 256QAM & 16QAM \\
\hline \hline
\end{tabular}




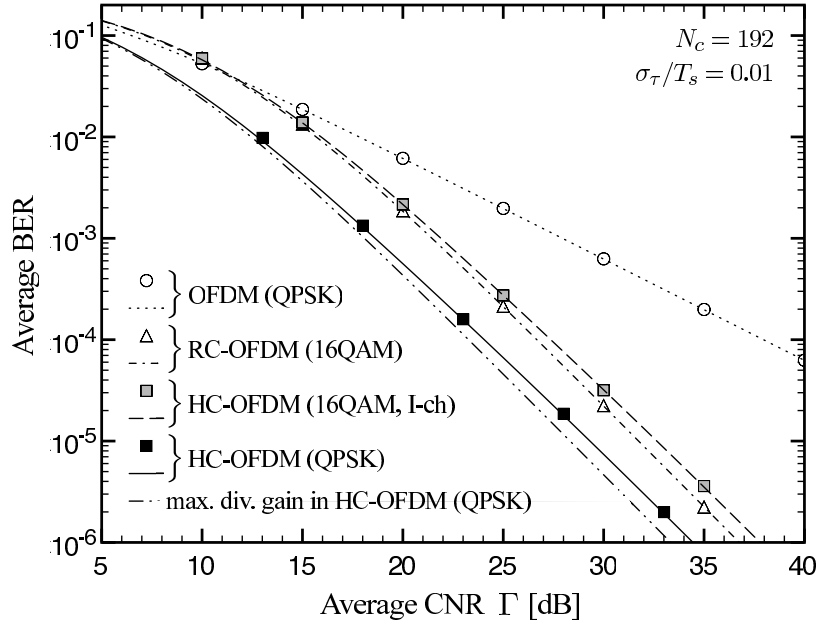

(a) average BER versus average $\mathrm{CNR}$

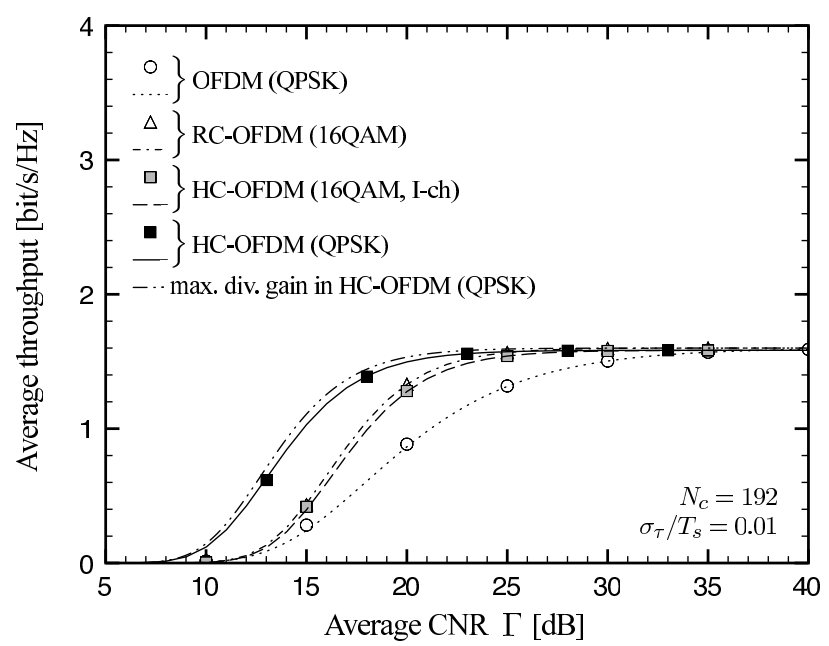

(b) average throughput versus average $\mathrm{CNR}$

Fig. 5 Performances over Rayleigh fading channels (case I, $N_{c}=192$, $\left.\sigma_{\tau} / T_{s}=0.01\right)$.

the RC-OFDM systems, because of less frequency diversity gain. But the HC-OFDM systems with both IQ channels can achieve the improvement of performances owing to the power efficiency in digital-modulation as compared with the RC-OFDM systems.

Figure 7 shows the average BER performance as a function of $\sigma_{\tau} / T_{s}$ when $N_{c}=192$ and $\Gamma=30 \mathrm{~dB}$. It is noted that the performance of the OFDM systems is irrelevant to the rms delay spread, then only analytical result is indicated in the figure. Moreover, the BERs with the maximum diversity gain in the RC-OFDM and HC-OFDM systems calculated by (13) are simultaneously indicated as reference. It is found from the figure that the HC-OFDM systems can outperform the conventional ones at any rms delay spread. The BERs in the RC-OFDM and HC-OFDM systems at $\sigma_{\tau} / T_{s}=0.002$ in Fig. 7(a) are close to each other, because the RC-OFDM systems have the advantages of frequency diversity gain. But, by comparing the BERs with the maximum diversity gain, the HC-OFDM systems have

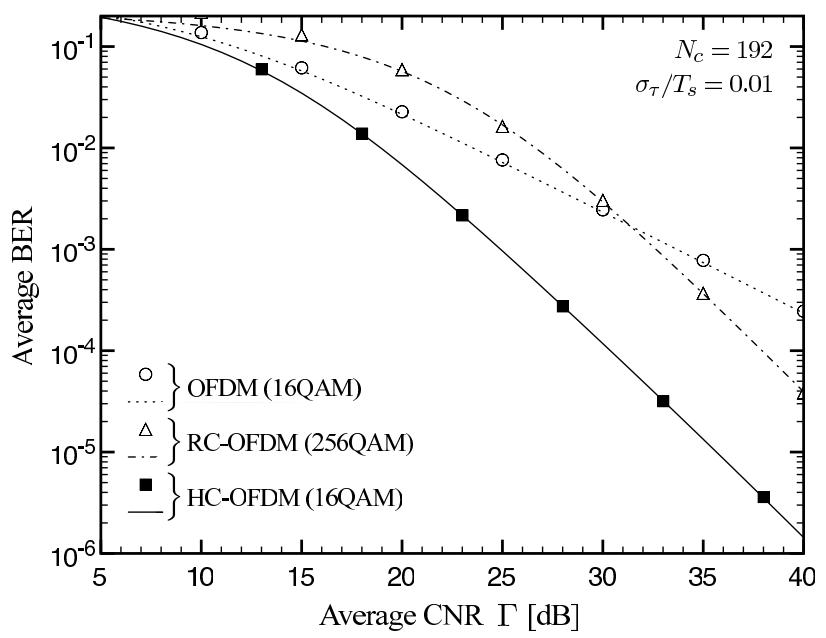

(a) average BER versus average $\mathrm{CNR}$

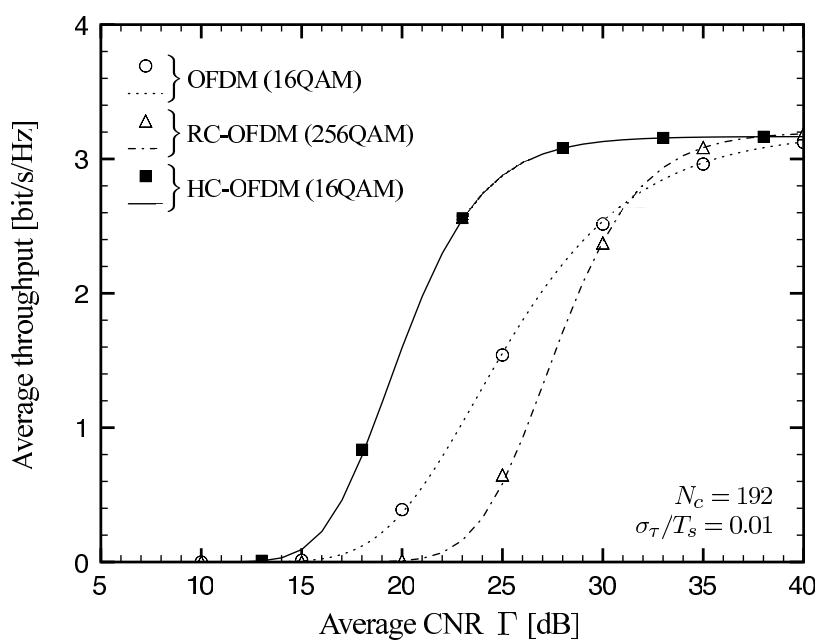

(b) average throughput versus average $\mathrm{CNR}$

Fig. 6 Performances over Rayleigh fading channels (case II, $N_{c}=192$, $\left.\sigma_{\tau} / T_{s}=0.01\right)$.

the advantage of power efficiency in digital-modulation.

Figure 8 shows the average BER and throughput performances of the HC-OFDM systems with 16QAM when $\sigma_{\tau} / T_{s}=0.005$ as parameter of subcarrier size $N_{c}$. Enlarging the size $N_{c}$ with the same OFDM symbol duration $T_{s}$ causes the OFDM bandwidth to broaden, which improves the performances close to the maximum ones by (13) and (14) because of increasing the frequency diversity gain.

Finally, we would like to emphasize in Figs. 4-8 that the simulation results confirm the theoretical performance analysis in Sect. 3.

\section{Performance with Turbo Coding}

As an application of the HC-OFDM systems in a practical situation, we briefly discuss the additional effect of errorcorrecting code by the simulation. Apart from the computational complexity shown in Fig. 2, the HC-OFDM systems can outperform the conventional ones as shown in the pre- 


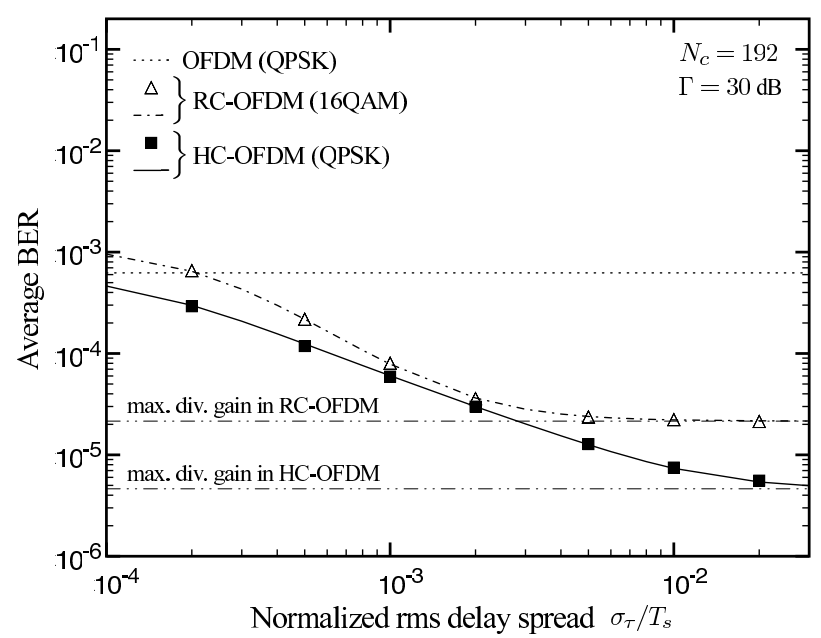

(a) case I

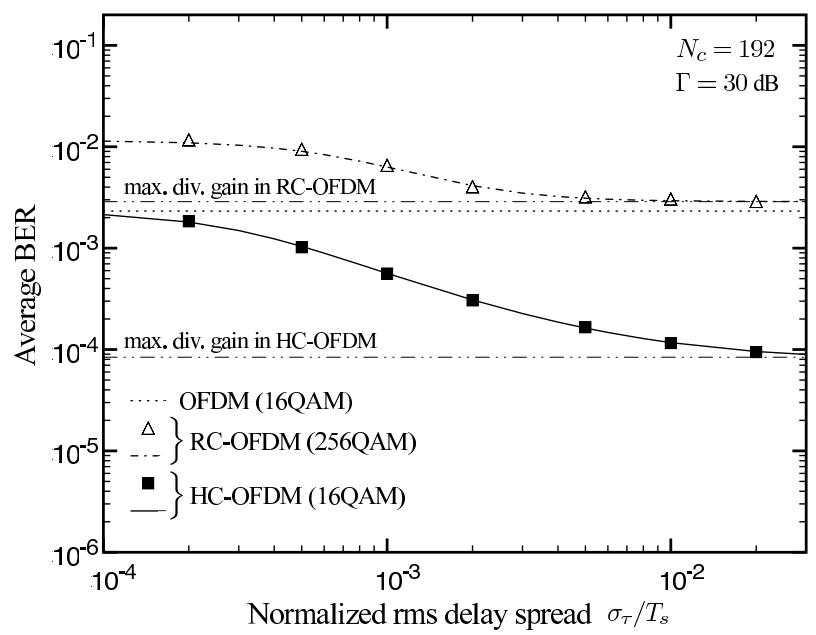

(b) case II

Fig. 7 Average BER performance as a function of normalized rms delay $\operatorname{spread}\left(N_{c}=192, \Gamma=30 \mathrm{~dB}\right)$.

vious section. Among them, the OFDM systems attain the worst performance on account of the lack of diversity gain, but the performance is expected to be drastically improved by the error-correcting code. Therefore, we will especially focus on the comparison of the OFDM systems including coding gain with the HC-OFDM systems including both diversity gain and coding gain in this section. We use popular rate-1/2 turbo code which consists of two recursive systematic convolutional encoders with constraint length of 3 concatenated in parallel. Generally, when the signal strength in an interleaved sequence fluctuates rapidly, the effect of coding gain is enlarged, then we will evaluate the performance in both fixed and mobile situations.

Figure 9 shows the average BER performance as parameter of the normalized maximum Doppler frequency $f_{D} T_{s}$ when $N_{c}=192, M_{t}=4$ and $\sigma_{\tau} / T_{s}=0.01$. The codeword is interleaved within 100 OFDM symbols and 192 subcarriers $(100 \times 192$ digital-modulated symbols $)$. It is found from the figure that the HC-OFDM systems largely outper-

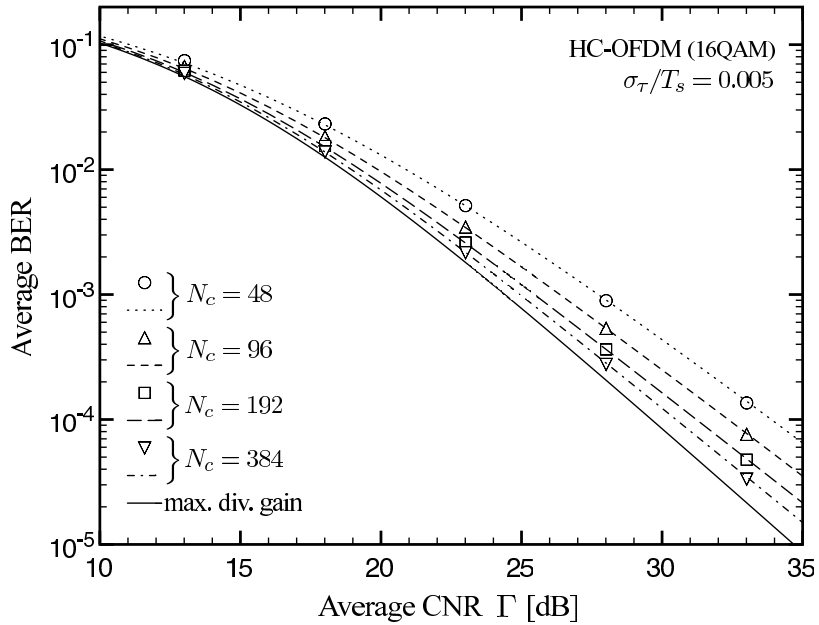

(a) average BER versus average $\mathrm{CNR}$

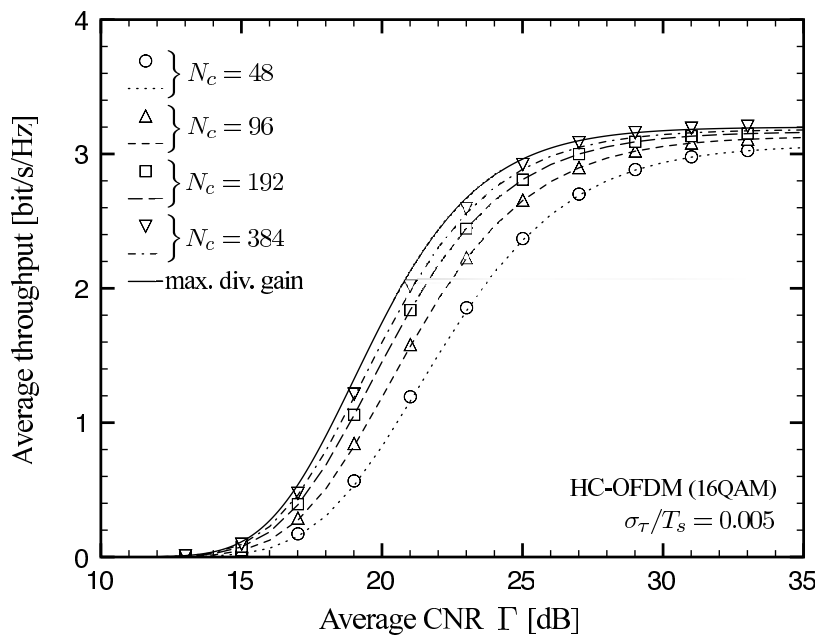

(b) average throughput versus average $\mathrm{CNR}$

Fig. 8 Performances of HC-OFDM with 16QAM $\left(\sigma_{\tau} / T_{s}=0.005\right)$.

form at low Doppler frequency, and that the performances of all systems are improved as the Doppler frequency becomes larger. The improvement is almost saturated when $f_{D} T_{s}=0.3$, and the HC-OFDM systems still have the advantage of about $0.8 \mathrm{~dB}$ at $\mathrm{BER}=10^{-5}$ over the OFDM systems because of the additional advantage of the frequency diversity gain. On the other hand, the performance gain against the RC-OFDM systems is always large on the channel condition as shown in Fig. 9 because of the power efficiency in digital-modulation.

Figure 10 shows the average throughput performance as parameter of the modulation level $M_{t}$ when $N_{c}=192$, $\sigma_{\tau} / T_{s}=0.01$ and $f_{D} T_{s}=0.1$. The HC-OFDM and RC-OFDM systems attain the same throughput in the region below $5.7 \mathrm{~dB}$ on the average $\mathrm{CNR}$, then the $\mathrm{HC}$ OFDM systems have the disadvantage that twice the modulation/demodulation processings and the phase compensator are necessary as shown in Fig. 2. But the HC-OFDM systems can outperform the RC-OFDM systems in the region above $5.7 \mathrm{~dB}$ for the same reason in Fig. 9. On the other 


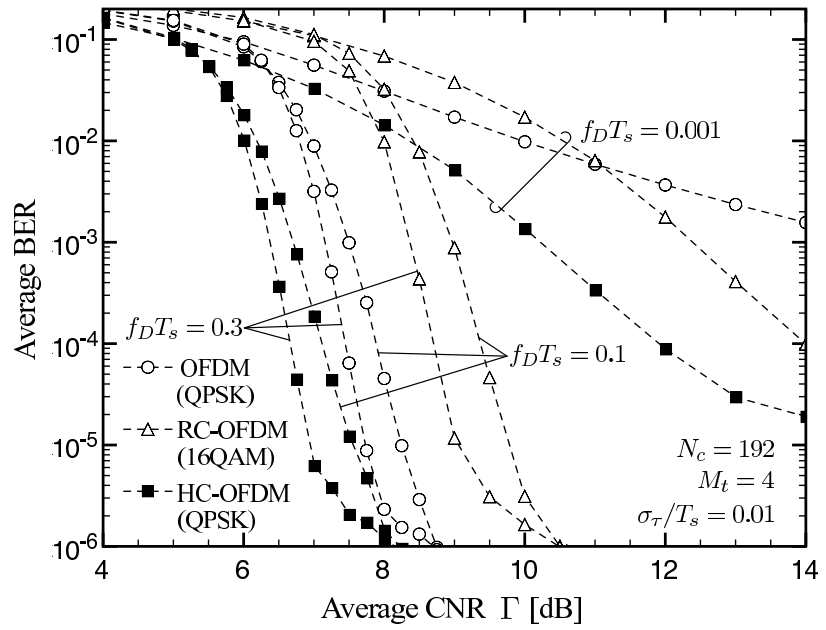

Fig. 9 Average BER performance of OFDM, RC-OFDM and HCOFDM with turbo coding $\left(N_{c}=192, M_{t}=4, \sigma_{\tau} / T_{s}=0.01\right)$.

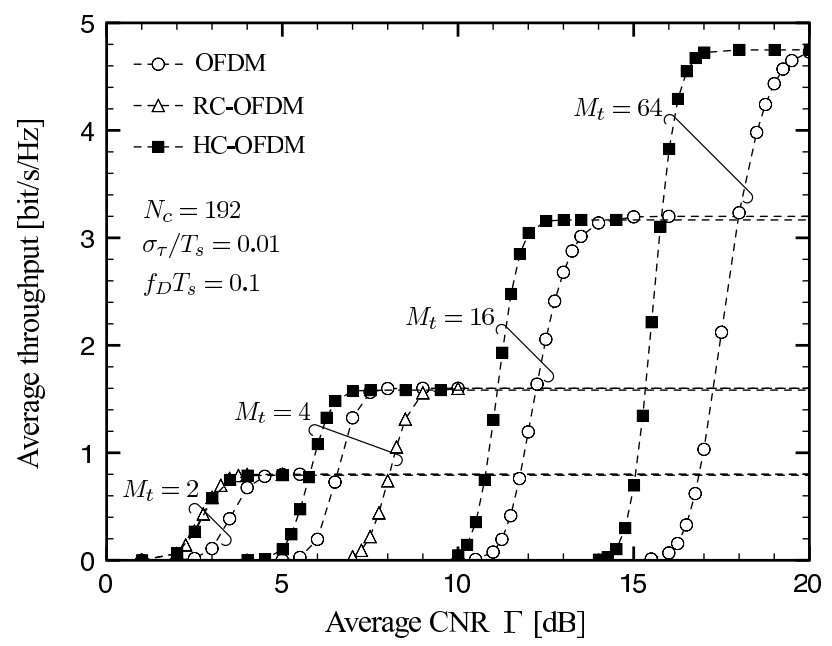

Fig. 10 Average throughput performance of OFDM, RC-OFDM and HC-OFDM with turbo coding $\left(N_{c}=192, \sigma_{\tau} / T_{s}=0.01, f_{D} T_{s}=0.1\right)$.

hand, the HC-OFDM systems attain the maximum throughput in lower required CNR at any modulation as compared with the OFDM systems. Therefore, the HC-OFDM systems have the advantage when applying the adaptive modulation.

\section{Conclusion}

In this paper, we derived closed-form equations for BER and throughput of wireless OFDM systems employing the Hermite-symmetric subcarrier coding. The subcarrier coding can force the imaginary part of the OFDM signal to be zero, then another data sequence can be simultaneously transmitted in the quadrature channel. Because of the simultaneous transmission of the two data sequences, higherlevel modulation to keep the same bit rate is unnecessary, while double carrier power is consumed. Therefore, the signal power per channel should be reduced by half in order to keep the same condition. But our analytical results theoretically indicated that the HC-OFDM systems achieve the improvement of BER and throughput performances despite half the carrier power and the imperfect diversity gain.

In order to evaluate the optimal effect of the Hermitesymmetric subcarrier coding, the perfect channel estimation is assumed for the purpose of ideal phase compensation and coherent detection in this paper. Actually, an accurate channel estimation is necessary for the HC-OFDM systems to completely separate IQ channels. Moreover, MIMO techniques are necessary to realize higher spectrum efficiency, for example, to cope with a rapid increase of smartphone or tablet in recent years. As future works, we would like to evaluate performances of the HC-OFDM systems considering the actual channel estimation and MIMO situation.

\section{Acknowledgement}

This work was supported by JSPS KAKENHI Grant Number 24560448 .

\section{References}

[1] S.B. Weinstein and P.M. Ebert, "Data transmission by frequencydivision multiplexing using the discrete Fourier transform," IEEE Trans. Commun., vol.COM-19, no.5, pp.628-634, Oct. 1971.

[2] J.A.C. Bingham, "Multicarrier modulation for data transmission: An idea whose time has come," IEEE Commun. Mag., vol.28, no.5, pp.5-14, May 1990.

[3] S.M. Alamouti, "A simple transmit diversity technique for wireless communications," IEEE J. Sel. Areas Commun., vol.16, no.8, pp.1451-1458, Oct. 1998.

[4] D. Agrawal, V. Tarokh, A. Naguib, and N. Seshadri, "Space-time coded OFDM for high data-rate wireless communication over wideband channels," Proc. IEEE VTC'98, vol.3, pp.2232-2236, May 1998.

[5] K.F. Lee and D.B. Williams, "A space-frequency transmitter diversity technique for OFDM systems," Proc. IEEE GLOBECOM'00, vol.3 pp.1473-1477, Nov. 2000.

[6] S. Ye, R.S. Blum, and L.J. Cimini, Jr., "Adaptive modulation for variable-rate OFDM systems with imperfect channel information," Proc. IEEE VTC Spring 2002, vol.2, pp.767-771, May 2002.

[7] T. Usui, F. Ishizu, and K. Murakami, "A study on adaptive modulation technique in OFDM," IEICE Trans. Commun. (Japanese Edition), vol.J88-B, no.3, pp.634-642, March 2005.

[8] Y.H. Kim, I. Song, H.G. Kim, T. Chang, and H.M. Kim, "Performance analysis of a coded OFDM system in time-varying multipath Rayleigh fading channels," IEEE Trans. Veh. Technol., vol.48, no.5, pp.1610-1615, Sept. 1999.

[9] J. Zheng and S.L. Miller, "Performance analysis of coded OFDM systems over frequency-selective fading channels," Proc. IEEE GLOBECOM2003, vol.3, pp.1623-1627, Dec. 2003.

[10] H. Futaki and T. Ohtsuki, "Performance of low-density paritycheck (LDPC) coded OFDM systems," Proc. IEEE ICC 2002, vol.3, pp.1696-1700, May 2002.

[11] I. Barhumi, G. Leus, and M. Moonen, "Optimal training sequences for channel estimation in MIMO OFDM systems in mobile wireless channels," 2002 International Zurich Seminar on Broadband Commun., Access, Transmission, Networking, pp.44-1-44-6, Feb. 2002.

[12] Y. Jing, D. Wang, M. Chen, S. Cheng, and H. Wang, "Reduced complexity MIMO-OFDM channel estimation based on parametric channel model," Proc. IEEE VTC2004-Fall, vol.2, pp.1372-1376, Sept. 2004. 
[13] W.Y. Zou and Y. Wu, “COFDM: An overview,” IEEE Trans. Broadcast., vol.41, no.1, pp.1-8, March 1995.

[14] IEEE P802.16-REVd - Standard for Local and Metropolitan Area Networks - Part 16: Air Interface for Fixed Broadband Wireless Access Systems.

[15] Y. Zhao and S.G. Haggman, "Intercarrier interference selfcancellation scheme for OFDM mobile communication systems," IEEE Trans. Commun., vol.49, no.7, pp.1185-1191, July 2001.

[16] F. Sasamori, Y. Ishikawa, S. Handa, and S. Oshita, "A simple expression of BER performance in COFDM systems over fading channels," IEICE Trans. Fundamentals, vol.E92-A, no.1, pp.332-336, Jan. 2009.

[17] Y. Ma, Y. Huang, F. Liu, and N. Yi, "Blind channel estimation for repetition coded OFDM in block Rayleigh fading," Proc. IEEE GLOBECOM2003, vol.1, pp.30-34, Dec. 2003.

[18] F. Sasamori, Z. Jia, S. Handa, and S. Oshita, "Performance analysis of repetition coded OFDM systems with diversity combining and higher-level modulation," IEICE Trans. Commun., vol.E94-B, no.1, pp.194-202, Jan. 2011.

[19] W. Matsumoto and H. Imai, "A study on half-symbol scheme of OFDM modulation and MC-CDMA modulation," IEICE Trans. Commun. (Japanese Edition), vol.J85-B, no.6, pp.910-921, June 2002.

[20] F. Maehara, F. Sasamori, and F. Takahata, "Inter-symbol interference suppression scheme using even-numbered sub-carriers for fixed-rate OFDM systems," IEICE Trans. Commun., vol.E87-B, no.4, pp.866872, April 2004.

[21] A. Fertner, M. Hyll, and A. Orling, "Computationally efficient analysis and synthesis of real signals using discrete fourier transforms and inverse discrete fourier transforms," U.S. Patent 6169723, 2 Jan. 2001.

[22] ANSI, Asymmetric Digital Subscriber Line (ADSL) Metallic Interface, ANSI Standard, T.413-1995.

[23] A. Batra, et al., "Multi-band OFDM Physical Layer Proposal for IEEE 802.15 Task Group 3a," IEEE P802.15-04/493r1-TG3a, Sept. 2004.

[24] A. Batra and J. Balakrishnan, "Improvements to the multi-band OFDM physical layer," IEEE Consumer Communications and Networking Conference, vol.2, pp.701-705, Jan. 2006.

[25] J. Tubbax, B. Come, L.V. der Perre, S. Donnay, M. Engels, H.D Man, and M. Moonen, "Compensation of IQ imbalance and phase noise in OFDM systems," IEEE Trans. Wireless Commun., vol.4, no.3, pp.872-877, May 2005.

[26] R. Corvaja and A.G. Armada, "Joint channel and phase noise compensation for OFDM in fast-fading multipath applicaitons," IEEE Trans. Veh. Technol., vol.58, no.2, pp.636-643, Feb. 2009.

[27] B. Yang, K.B. Letaief, R.S. Cheng, and Z. Cao, "Channel estimation for OFDM transmission in multipath fading channels based on parametric channel modeling," IEEE Trans. Commun., vol.49, no.3, pp.467-479, March 2001.

[28] Y. Karasawa, Radiowave Propagation Fundamentals for Digital Mobile Communications, Corona Publishing, Tokyo, 2003 (in Japanese).

[29] P.A. Bello, "Characterization of randomly time-variant linear channels," IEEE Trans. Commun. Syst., vol.CS-11, no.4, pp.360-393, Dec. 1963.

[30] W.C. Jakes, Microwave Mobile Communications, IEEE Press, New York, 1974.

[31] M. Patzold and A. Szczepanski, "Methods for modeling of specified and measured multipath power delay profiles," Proc. IEEE VTC2000-Spring, vol.3, pp.1828-1834, May 2000.

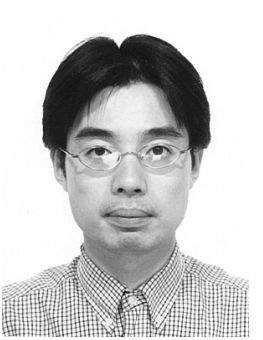

Fumihito Sasamori received the B.E., M.E. and Dr. Eng. Degrees from Waseda University, Tokyo in 1994, 1996 and 2000, respectively. Since 2000 he has been with the Department of Electrical and Electronic Engineering, Shinshu University, first as a Research Associate and since 2006 as an Associate Professor. His current research interests include digital mobile communication systems. He received the IEICE Young Engineer Award in 2000. He is a member of IEEE.

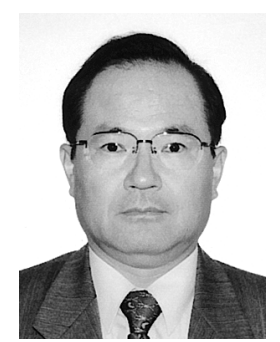

Shiro Handa received the B.E. and M.E. degrees from Shinshu University in 1978 and 1980 respectively, and the Dr. Eng. degree from Kobe University in 1988. From 1982 to 1988, he was a Research Associate at Kobe University. From 1988 to 1994, he was with Nagano National College of Technology. He has been with the Department of Electrical and Electronic Engineering, Shinshu University, since 1994 as an Associate Professor and since 2005 as a Professor. In 1996, he was at the University of California, Davis, as a visiting researcher. His research interests include satellite and mobile communication systems, modulation and coding, and data compression. He is a member of IEEE and SITA. 